\title{
Aspectos biofísicos y socioeconómicos de la subcuenca del rio Páez, Cartago, Costa Rica
}

\author{
Biophysical and socioeconomic aspects of the Páez river \\ sub-basin, Cartago, Costa Rica.
}

\author{
María Álvarez-Jiménez ${ }^{1}$ \\ Universidad Nacional, Costa Rica, Costa Rica \\ Pablo Ramírez-Granados ${ }^{2}$ \\ Universidad Nacional, Costa Rica, Costa Rica \\ José Castro-Solis ${ }^{3}$ \\ Universidad Nacional, Costa Rica, Costa Rica
}

\section{Resumen}

El estudio consistió en una caracterización de variables biofísicas y socioeconómicas de la subcuenca del rio Páez, Cartago con el propósito de generar una línea base de la cuenca en el sector norte de Cartago para la toma decisiones por parte de diferentes actores y para proponer lineamientos de manejo de recursos hídricos en un eventual manejo. Se utilizó técnicas de investigación cuantitativas y cualitativas entre ellos la aplicación de una encuesta a 178 personas en 10 poblados de la subcuenca distribuidos en la parte alta, media y baja. La subcuenca tiene un área de $28,34 \mathrm{~km}^{2}$, se ubica en los cantones de Oreamuno y Paraíso aproximadamente más del 50\% del territorio se dedica a actividades agropecuarias, un $16 \%$ posee cobertura forestal como los usos más importantes, presenta problemáticas importantes de vulnerabilidad, contaminación ambiental, gobernabilidad,

1 MSc. María Álvarez Jiménez. Laboratorio de Hidrogeología y Manejo de Recursos Hídricos. Escuela de Ciencias Ambientales, Universidad Nacional: maria.alvarez.jimenez@una.cr.

(D) https://orcid.org/0000-0002-5266-4496

2 MSc. Pablo Ramírez Granados. Laboratorio de Hidrogeología y Manejo de Recursos Hídricos. Escuela de Ciencias Ambientales, Universidad Nacional: pablo.ramirez.granados@una.cr.

(iD https://orcid.org/0000-0002-5082-445X

3 Consultor, MSc. José Castro Solís. Laboratorio de Hidrogeología y Manejo de Recursos Hídricos. Escuela de Ciencias Ambientales, Universidad Nacional: jose.castro.solis@una.cr

(iD https://orcid.org/0000-0002-3393-6951 
diversificación de opciones de empleo en la zona. Se evidencia el papel que cumple la subcuenca del rio Páez como parte fundamental para el abastecimiento agua potable y el desarrollo de actividades productivas, la caracterización planteada brinda una base para determinar el estilo de manejo que requiere la cuenca.

Palabras clave: aspectos biofísicos, socioeconómicos, cuenca Páez.

\begin{abstract}
The present study consisted in a characterization of biophysical and socioeconomic variables of the Páez River sub-basin, located in the norther sector of the city of Cartago, Costa Rica, with the purpose of generating baseline knowledge aimed at facilitating actors with decision-making tools and to propose water resource management guidelines for their eventual implementation. Quantitative and qualitative research techniques were used, including the application of a survey to 178 people in 10 localities geographically distributed in the upper, middle and lower sections of the sub-basin. The Páez River sub-basin covers an area of $28.34 \mathrm{~km}^{2}$ in the cantons of Oreamuno and Paraíso and over $50 \%$ of the territory is dedicated to agricultural activities and $16 \%$ to forest coverage. This geographical region presents important problems such as climatic vulnerability environmental pollution, governance issues, deficient diversification of economic activities, and thus poor diversity of employment opportunities in the region. The role of the Páez River sub-basin as a fundamental component for the supply of potable water and water for the development of productive activities is evidenced, the proposed characterization provides a baseline for determining adequate basin management methodologies.
\end{abstract}

Keywords: Biophysical and socioeconomic aspects; Páez River sub-basin.

\title{
Introducción
}

Una forma de entender cómo las sociedades humanas deterioran el ambiente natural del cual dependen es tratando de encontrar dónde las actividades socioeconómicas se relacionan con el ambiente biofísico (Coronel et al; 2012). Parte del deterioro se debe a un acelerado crecimiento de la población sin una adecuada planificación y ordenamiento territorial en las cuencas hidrográficas desencadenando problemáticas.

Lange et al; (2010), citado por Pirani y Mousavi, (2016) coincide que dichas problemáticas están relacionadas con la planificación en las cuencas y ocurren debido a que han ignorado las condiciones socioeconómicas. Igualmente, Elizondo (2013) plantea la necesidad de conocer la estructura económica para comprender las decisiones de manejo en la cuenca.

Estratégicamente el planteamiento de problemas y soluciones en las cuencas debe enfocarse a partir de la correlación existente entre la unidad de manejo y las actividades que las personas desarrollan en ella (Villareal, 2007), esto referido a aspectos biofísicos y socioeconómicos. Estas variables generan información base y son una herramienta para realizar 
evaluaciones de los cambios en la cuenca a través de indicadores de impacto (Carrie, 2004), que posteriormente pueden funcionar para toma de decisiones en el manejo de la cuenca.

El presente artículo se refiere a la subcuenca del río Páez; ya que es considerada como una de las más importantes del sector central de Cartago por formar parte de la cuenca del rio Reventazón. Se distingue por ser una zona de producción agropecuaria y energía hidroeléctrica de Costa Rica, además este sitio posee manantiales, quebradas y ríos que son aprovechados por las comunidades de Oreamuno y Paraíso para consumo humano y desarrollo de actividades productivas.

A su vez presenta múltiples problemas ambientales relacionados con malas prácticas del uso del suelo, vulnerabilidad y riesgos, inadecuado manejo residuos sólidos, contaminación de los ríos; sin embargo, hasta la fecha la generación de investigación ha sido limitada en la subcuenca, y no se cuenta con información biofísica y socioeconómica necesaria para tener una línea base.

De acuerdo con Siles \& Soares (2003) la cuenca hidrográfica interactúa de manera dinámica con sistemas socioeconómicos y biofísicos y en ella se refleja un comportamiento que responde a estilos de manejo de los recursos agua, suelo, flora y fauna, así como las actividades o infraestructuras existentes en su zona de influencia. Por tanto, la determinación de dichos aspectos en la subcuenca del rio Páez es necesaria destacar las potencialidades y las limitaciones que tienen los recursos y así poder bridar un adecuado manejo y soluciones a problemáticas identificadas.

\section{Área de Estudio}

La subcuenca del río Páez se ubica en la zona noroeste de la cuenca del río Reventazón, al norte se encuentra dentro del área de conservación Parque Nacional Volcán Irazú y al sur con el cantón de Paraíso. Entre las coordenadas planas 515682 y 519780 m este y 1102192 y 1087436 m norte del sistema de Proyección Transversal de Mercator para Costa Rica y ubicada en la zona central de la hoja cartográfica nacional Istarú (1:50 000) (Alfaro, 2018).

Cuenta con un área de 28,34 $\mathrm{km}^{2}$ y se ubica en los cantones de Oreamuno y Paraíso de la provincia de Cartago (Figura 1), abarca los pueblos 
Pisco, Cot, Paso Ancho, Páez, San Juan de Chicúa, Cuesta Los Quemados, Paraíso, Finca Ivancovich, Rincón, Alto Birrisito y Ujarrás.

Figura 1. Ubicación geográfica de la subcuenca del río Páez

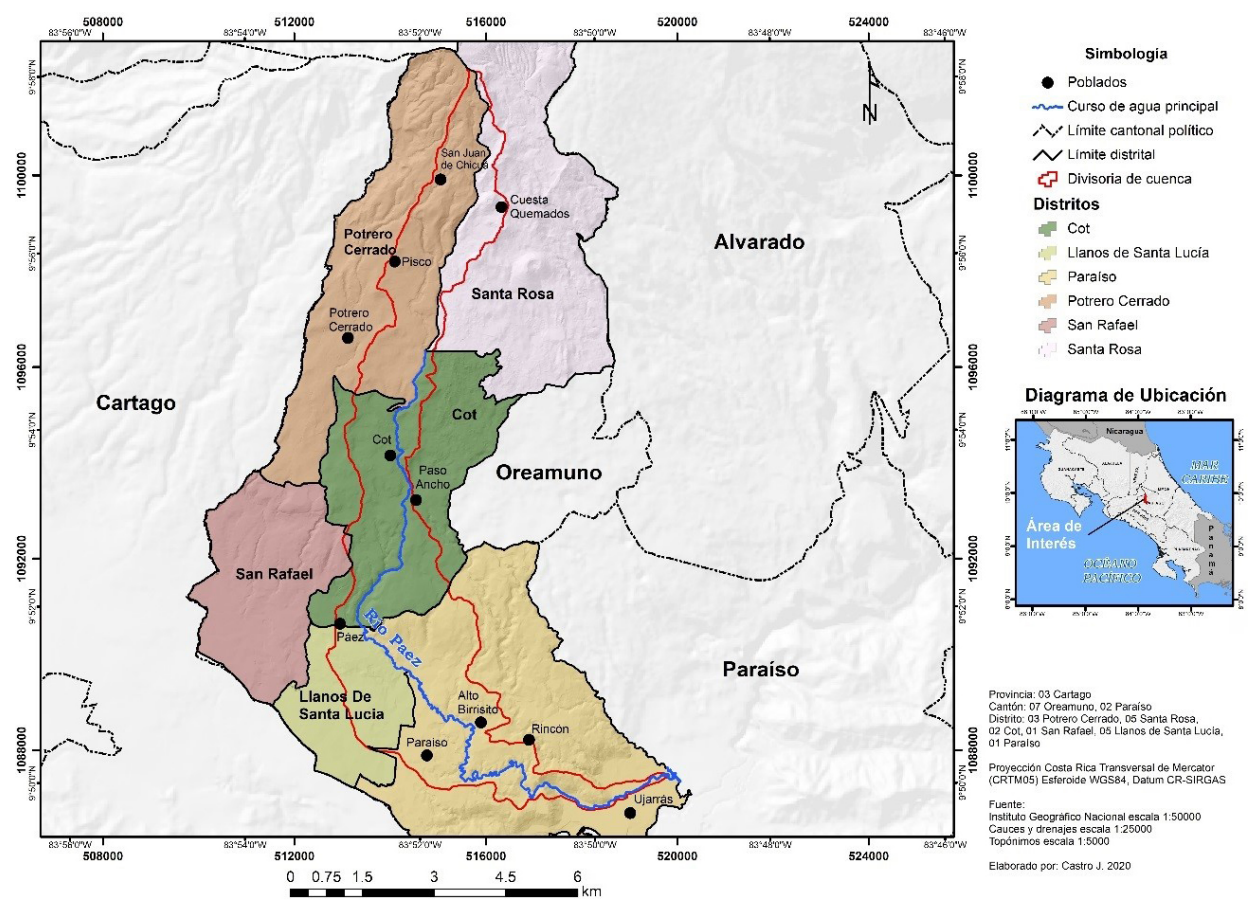

Fuente: Castro, 2020

\section{Metodología}

Para la determinación de las variables biofísicas y socioeconómicas, se utilizó como base la metodología (Watler et ál., 2013, p. 8) y se indago fuentes de información secundaria y primaria obtenida en campo. Se realizó visitas y consultas claves a personas de instituciones estatales, municipios de la región, y se generó mapas utilizando la herramienta ArcGIS.

Para determinar las variables biofísicas se consideró el área total de la subcuenca, precipitación, hidrográfica, fisiografía, topografía, geomorfología, geología, tipo de suelo, uso de la tierra, amenazas, zonas de vida, biodiversidad. En cuanto a las variables socioeconómicas; la demografía, salud, educación, vivienda, servicios públicos, infraestructura vial, 
transporte, uso del agua y contaminación, actividades productivas, empleo, gobernabilidad.

Se aplicó una encuesta para obtener información y se realizó un muestreo a 178 personas en los 10 pueblos ubicados en la parte alta, media y baja de la subcuenca, a través de un muestreo, la estimación del tamaño de la muestra se realizó a partir de la aplicación de la fórmula para el tamaño de muestra en poblaciones infinitas, utilizando un nivel de confianza de $90 \%$ y un error de estimación del $6 \%$. Se estratificó tomando en cuenta el número de familias en cada comunidad de estudio y entrevisto solamente una persona por familia mayor a 18 años.

Se obtuvo información climática proveniente de registros de estaciones meteorológicas del Instituto Meteorológico Nacional (IMN); San Juan de Chicúa, Volcán Irazú, Potrero Cerrado, Dulce Nombre, Instituto Tecnológico de Costa Rica, Paraíso y Cachi Plantel. Se determinó la precipitación zonal a través del método de polígonos de Thiessen y la para la determinación de los órdenes de los drenajes se utilizó el método de Strahler (1957).

\section{Resultados}

La parte alta de la subcuenca presenta precipitaciones mayores promedio de $3090 \mathrm{~mm}$, seguido con $2225 \mathrm{~mm}$ en la parte media y en la parte baja con $1360 \mathrm{~mm}$, respectivamente. La zona de estudio tiene rangos de precipitación media que representa variabilidad desde la parte alta hasta la parte baja (Figura 2). Posee tres zonas de vida; Bosque Húmedo Montano bajo con un rango de precipitación anual es de $1400 \mathrm{~mm}$ a $2700 \mathrm{~mm}$, con una biotemperatura media que varía entre $18^{\circ} \mathrm{C}$ y $12^{\circ} \mathrm{C}$, Bosque Húmedo Premontano con un rango de precipitación 700-1400 mm , presentando una biotemperatura de $24{ }^{\circ} \mathrm{C}$ y $18{ }^{\circ} \mathrm{C}$ y Bosque Muy Húmedo Montano con un rango de precipitación de 2400 a $3700 \mathrm{~mm}$ y una biotemperatura de $12{ }^{\circ} \mathrm{C}$ y $6{ }^{\circ} \mathrm{C}$ (Quesada, 2007). 
Figura 2. Distribución de la precipitación de la subcuenca del río Páez, Cartago

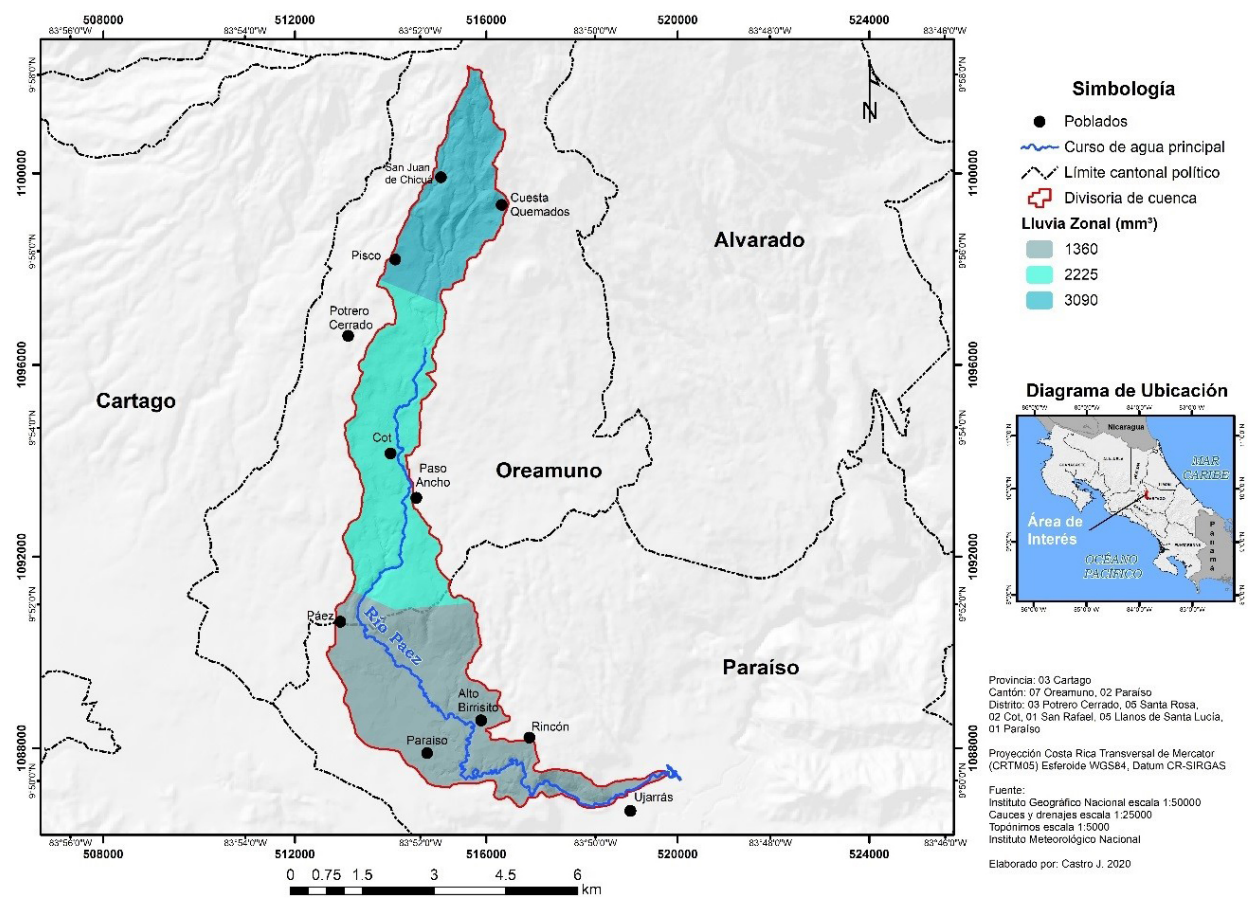

Fuente: Castro, 2020

Respecto a las variables hidrológica; el rio Páez forma parte de las 11 subcuencas que pertenecen al río Reventazón (Campos, 2010). La subcuenca es drenada por el río del mismo nombre, constituido por la quebrada Cañada, quebrada Paso Real, quebrada Pasquí y el río Quemados. Las quebradas tributarias son Paquín y Pollo, el nacimiento del río Páez se encuentra entre la finca la Cañada y el cerro Guardián, con un parámetro de drenaje que tiene la subcuenca paralela, y desemboca en la laguna de Cachí (Chin et al., 2016). El sistema fluvial del cantón de Oreamuno en la hoja topográfica Istaru del Instituto Geográfico Nacional (IGN), corresponde a las subvertientes Caribe y norte de la vertiente del Caribe. A la subvertiente norte corresponde la cuenca del río Reventazón-Parismina, es irrigada por los ríos Reventado, Retes, Yerbabuena, Chinchilla, San Nicolás, Tatiscú, Páez, Birrisito, Birrís, y la quebrada Pacayas; los cuales nacen en el cantón en la ladera sur del volcán Irazú 
De acuerdo con Arias (1987), el río Páez está compuesto por dos captaciones a manantiales: Paso Ancho y Lankaster, es una fuente importante de agua potable para abastecer sectores de San Rafael de Oreamuno y la ciudad de Cartago, también las aguas de las subcuencas Birris y Páez son aprovechadas por JASEC para generar energía eléctrica y abastecer los cantones de Alvarado, Oreamuno, Paraíso, Cartago y el Guarco. Se abastece de agua potable las poblaciones de Pacayas, Cot, Paso Ancho, Boquerón, Cipreses, San Pablo, Santa Rosa, Irazú Sur, La Pastora, San Juan de Chicuá, Potrero Cerrado, Capellades y Cervantes, por medio de manantiales, lo cual favorece el desarrollo de las comunidades y las actividades agrícolas, pecuarias e industriales. Para el país, esta zona genera gran parte de la producción hidroeléctrica, productos agrícolas, leche y carne; además de su gran belleza escénica para el turismo (Artavia, 2017).

La subcuenca del río Páez se encuentra sobre el acuífero Cartago, el cual tiene un caudal promedio que oscila entre $1 \mathrm{~L} / \mathrm{s}$ a $15 \mathrm{~L} / \mathrm{s}$, y un espesor de $65 \mathrm{~m}$ (Rojas, 2011). En la parte media de la subcuenca se encuentran las nacientes Ivancovich I e Ivancovich II, Lankester y Paso Ancho (Chin, Aguilar, Vega, y Espinoza, 2016).

El parámetro de orden máximo de los drenajes se refiere a la estructura de la red, y en la subcuenca del río Páez es de 5 (Figura 3), la mayoría de ordenes corresponde a orden 1 seguidos de los de orden 2, en la parte alta tienen a ser más numerosos los órdenes, y Alfaro (2018), plantea que el sistema de drenaje en este sector posee una estructura más compleja comparada con la sección media y baja, y genera una respuesta más rápida a drenar el agua de escorrentía, mostrando un mayor potencial de generar altos volúmenes de escorrentía en el cauce en instantes. 
Figura 3. Hidrografía de la subcuenca del río Páez. Cartago.

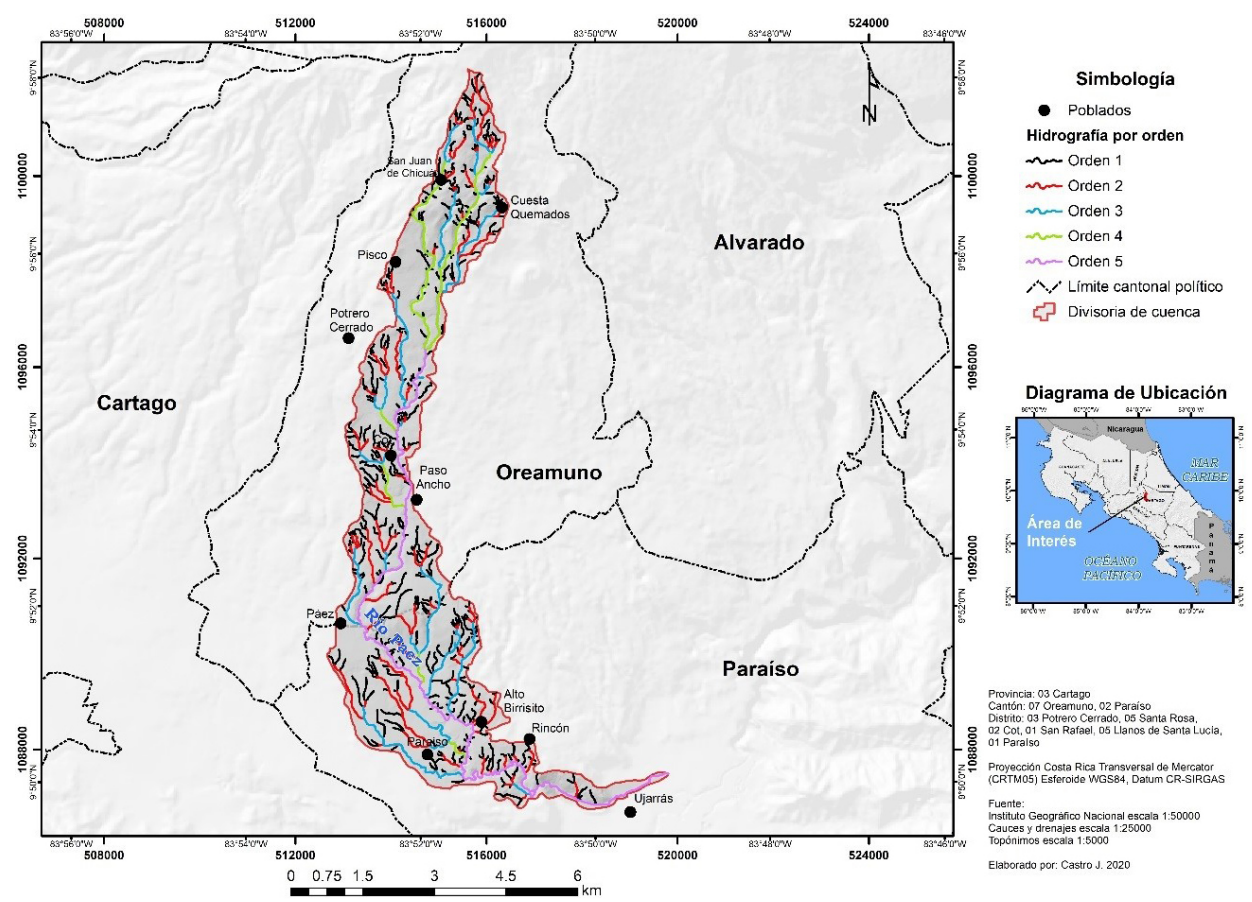

Fuente: Castro, 2020.

En relación con las características fisiográficas, posee un rango altitudinal que oscila entre 800 a 3400 m.s.n.m., la parte alta y media presenta un relieve montañoso formado por dos cordilleras: la Central en donde se encuentra el Volcán Irazú, el más alto del país y la cordillera de Talamanca. Según Instituto de Desarrollo Rural [INDER] (2016), el relieve de la parte baja tiene terrenos con altitudes menores; en esa sección se ubican el distrito de Cachi con pendientes planas y moderadas.

Las características topográficas, posee un rango de porcentaje de pendiente de $<15 \%$ a $>30 \%$, en la parte alta corresponde a pendientes fuertes y escarpados mayores a 30\%, en ella se ubican los poblados de San Juan de Chicuá, Cuesta Los Quemados y Pisco, Alto Birrisito, Páez, laderas del Volcán Irazú (Figura 4), en este sector se desarrollan actividades agrícolas y ganaderas en pendientes muy pronunciadas y con prácticas no adecuadas lo que provoca problemas de erosión. 
En la parte media y baja presenta la categoría de plano a moderadamente ondulado ( $0 \%-15 \%)$; y en ella se ubican la mayor cantidad de asentamientos urbanos pertenecientes al distrito de Paraíso y parte de Cot. Así mismo, en estos sectores se ubican poblados, que en algunos casos son vulnerables por encontrarse en sitios con pendientes fuertes.

Figura 4. Pendientes de la subcuenca del río Páez, Cartago.

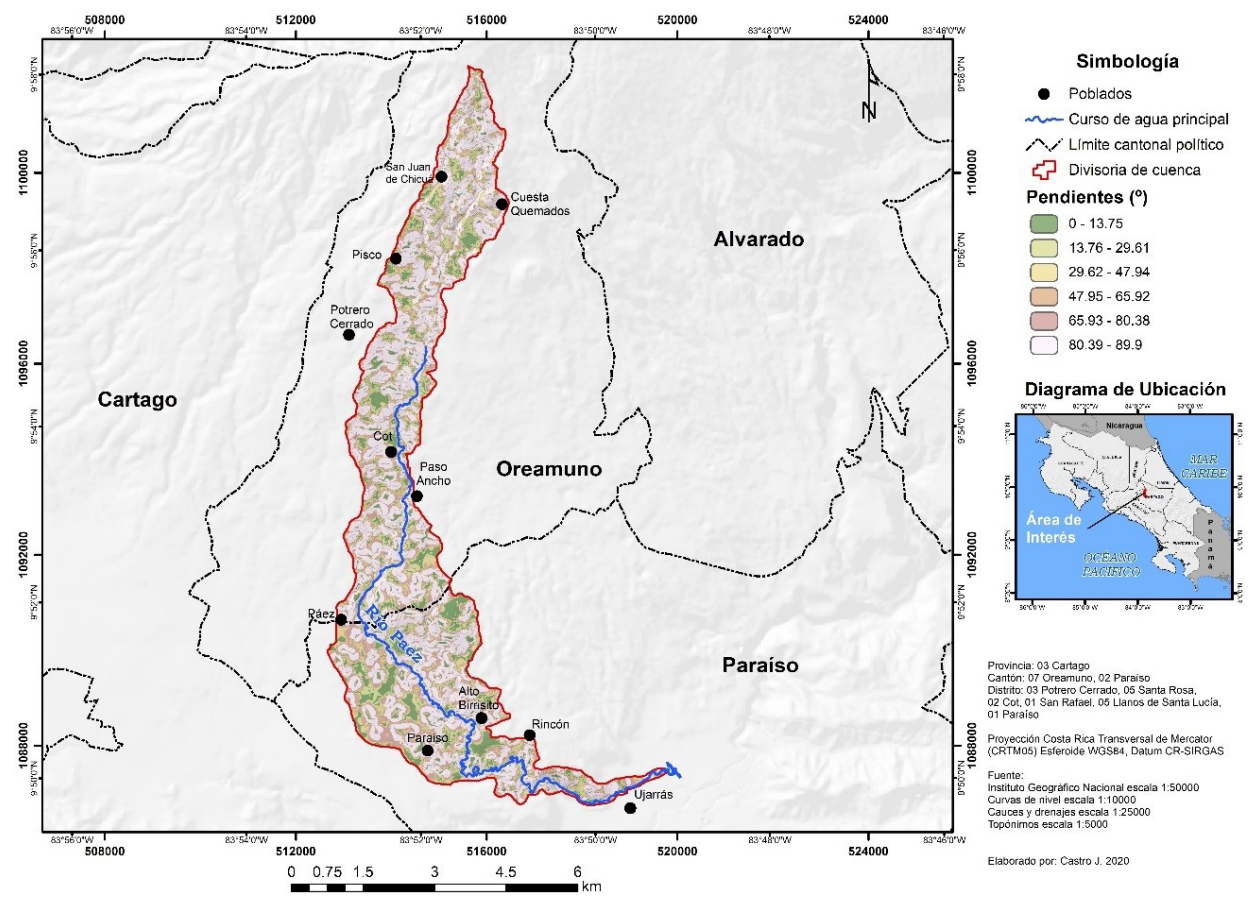

Fuente: Castro, 2020.

Respecto a la geomorfología y geología presenta que las estribaciones del volcán Irazú son el resultado del acúmulo de rocas volcánicas, depósitos de ceniza, lapilli y de flujos de lava. Hacia el oeste, en el cruce del Cristo afloran los depósitos de lahares provenientes del volcán Irazú, dentro de la zona de Paso Ancho se presentan depósitos de lahares en el cauce del río. En la misma zona y cercanías a la región de Boquerón se logra visualizar a nivel superficial la colada de Cervantes. La región central de la subcuenca se caracteriza por ser una meseta de ceniza, hacia la región este se encuentra la colada de Cervantes (Salazar, 2015, citado por Chin et al. 2016). 
Los flujos de lava muestran espesores de decenas de metros y fracturas columnares. Los lahares poseen espesores métricos, son suaves y fácilmente erosionables, presentan bloques subredondeados, bloques angulares y con alta variabilidad en tamaño, que van desde diámetros milimétricos a métricos, dentro de una matriz arcillosa. Las capas de ceniza varían de espesores métricos dependiendo de su fuente, con espesores mayores en los flancos noroeste y norte, y menores hacia el oeste, sur y este (Chin et al., 2016).

A nivel regional, la subcuenca del río Páez presenta unidades geológicas importantes distribuidas en la parte alta, media y baja, denominadas; la unidad Sapper, la unidad Reventado y la colada de Cervantes.

De acuerdo con Alvarado y Vega (2013), la colada de Cervantes presenta un incipiente desarrollo de hidrológico donde se presentan pocas quebradas sin nombre, de escasa longitud y caudal, solo presente en épocas de lluvias debido a la elevada permeabilidad del terreno. Esta unidad morfológica está delimita por cauces principales y desarrollados como los ríos Paso Real y Páez en la sección oeste, el río Birrís hacia el este y el Reventazón en su parte frontal.

La unidad Reventado se refiere a las rocas volcánicas de finales del Pleistoceno que han sido divididas cronológicamente en Miembro Paraíso, Miembro Cama de ceniza y Miembro Superior y cuya localidad tipo se encuentra en el cañón del río Reventado (Krushensky, 1972). Dichas formaciones corresponden a un $87.21 \%$ del área total de la subcuenca.

La secuencia de 15 o más flujos de lava, lahares intercalados y lechos de ceniza que se superponen incondicionalmente a la Formación Reventado en el área de las cabeceras del Río Reventado se llama la Formación Sapper. El nombre está tomado del pico acantilado $1.7 \mathrm{~km}$ al suroeste del cráter activo de Volcán Irazú, donde un espesor la secuencia de la formación está expuesta. La Formación Sapper es diferenciado de la Formación Reventado principalmente sobre la base de flujos de lava distintivos y en el contacto diferenciado con la Formación Reventado (Krushensky, 1972).

La unidad geológica denominada Formación Sapper corresponde a un $2.87 \%$ del total del área en estudio, y de acuerdo con Arellano et al. (2012) refiere a una secuencia de 15 o más flujos de lavas, intercalados con lahares y capas de cenizas que sobreyacen de forma discordante a la Formación Reventado, en las partes altas de la cuenca del río Reventado. Las coladas de lava de la Formación Sapper se caracterizan por ser densas, oscuras y negro verdosas a negras. 
De acuerdo con la geología específica del sito de estudio, la naciente Los Quemados ubicada en Potrero Cerrado, según Arellano et al. (2012) está compuesta por varios brotes de agua que ocurren en una zona inestable dentro del cauce del río Quemados a unos $400 \mathrm{~m}$ aguas abajo del puente sobre la carretera entre San Juan de Chicuá y La Pastora, ubicada en la parte alta de la zona de estudio, específicamente en el suroeste del Cerro Gurdián y cuenta con 3 unidades litológicas aflorando.

Respecto al tipo de suelo que presenta la subcuenca, (Bergoeing y Malavassi 1982, Víquez 1984, citado por Ramírez et al; 2008) plantean que los suelos de la región han sido influenciados por el aporte de ceniza volcánica y brechas y fragmentos de lava, dada la cercanía del área de estudio al cráter del volcán Irazú. Esta área se desarrollan actividades agropecuarias, como los cultivos de hortalizas, tubérculos, flores y la ganadería y presenta cuatro tipos de suelo; entisoles que se distribuyen en la parte alta, los andisoles ubicados en la parte media y alta, ultisoles y los inceptisoles en la parte baja respectivamente (Figura 5).

Figura 5. Ordenes de suelo, subcuenca Páez, Cartago

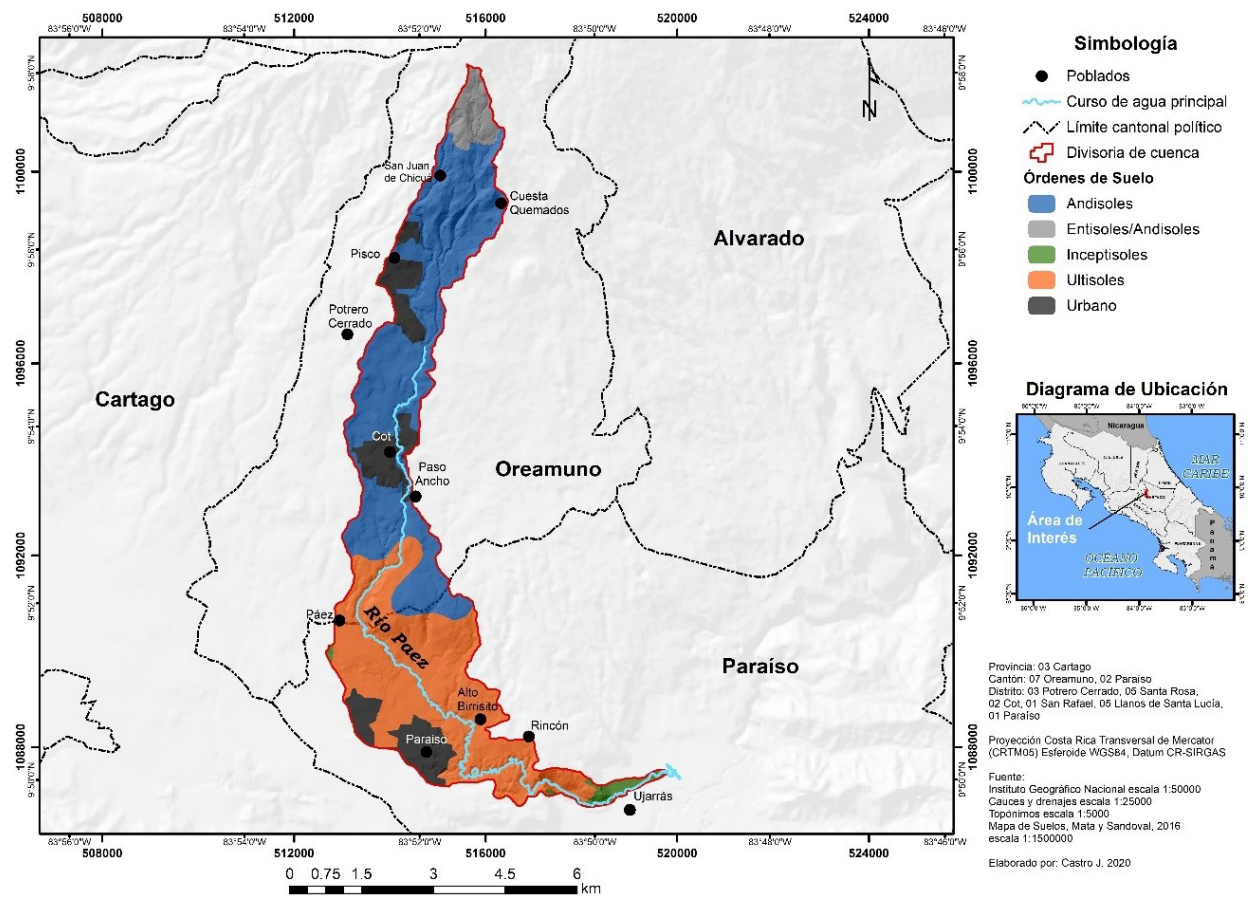

Fuente: Castro, 2020. 


\section{Uso del suelo y desarrollo de actividades productivas}

El uso que más prevalece en la subcuenca son las tierras agrícolas y pastos corresponden, conforman más del $50 \%$ del territorio (Cuadro 1). En la parte alta y media de la cuenca, en Oreamuno (Cot, Potrero Cerrado, Santa Rosa) se dedica al cultivo de papa, zanahoria, cebolla, coliflor, brócoli, repollo, remolacha. De acuerdo con SEPSA (2018) en el periodo 2017 -2018 este sector tuvo la mayor área sembrada de repollo, también es uno de los principales sitios productores de cebolla en Costa Rica por poseer grandes extensiones de tierra dedicadas a ese cultivo. En la parte baja en Paraíso (Cachi y Ujarrás) se produce chayote, tomate, chile, vainica, café, flores, helechos y algunos cultivos orgánicos en menor grado.

Por el gran desarrollo de esta actividad es una fuente de ingresos que genera empleo en la subcuenca, sin embargo, el 51\% de la población encuestada afirma que esta actividad no cuenta con prácticas ambientales sostenibles por el uso de agroquímicos en los cultivos que pueden afectar la salud de las personas y consideran no tener un cambio de cultura en sus prácticas.

Una problemática asociada a esta actividad el cambio de uso del suelo en las partes alta de pastos a cultivos en pendientes pronunciadas provocando problemas de erosión, según Programa de Investigación en Desarrollo Urbano Sostenible [PRODUS] (2016) no se aplican técnicas de conservación de suelos en las fincas agropecuarias lo que aumenta el problema.

La ganadería es otra actividad desarrollada en este sector por tener suelos fértiles y aptos para ganadería de leche, carne y doble propósito.

Cuadro 1. Área absoluta y porcentual del uso del suelo de la subcuenca del río Páez, Cartago.

\begin{tabular}{|c|c|c|}
\hline Uso de la tierra & Área $\mathbf{( K m}^{\mathbf{}} \mathbf{)}$ & Área (\%) \\
\hline Bosque & 4.34 & 15.29 \\
\hline Urbano & 3.97 & 13.98 \\
\hline Agropecuario-pasto-cultivo & 19.37 & 68.23 \\
\hline Cultivo Permanente & 0.71 & 2.50 \\
\hline Total & 28.4 & 100 \\
\hline
\end{tabular}

Fuente: Elaboración propia.

La cobertura forestal es el segundo uso con $15.29 \%$, se concentra en las partes altas de la zona de estudio. De acuerdo con Arias-Cascante 
(2018) existen 17 contratos destinados al Pago por Servicios Ambientales (protección de bosque, recurso hídrico, vacíos de conservación y sistemas agroforestal), ubicados en principalmente en Oreamuno y Paraíso.

Ese sistema de protección forestal beneficia la captación hídrica necesaria para las poblaciones de la subcuenca del río Páez. Además, es una zona Cot y Potrero Cerrado, (parte alta y media) donde se desarrolla el turismo y es una fuente de ingresos para los habitantes, allí se encuentra el Parque Nacional Volcán Irazú (PNVI) en la parte alta y ocupa el tercer lugar entre las ASP del SINAC por número total de visitantes.

Luego el uso urbano representa el 14\%, ubicado en la parte baja de la cuenca en su mayoría (Paraíso y parte de Oreamuno), ha cambiado el paisaje de manera drástica y el estado de los recursos de la cuenca (Figura 6).

Figura 6. Uso actual de la tierra en la subcuenca del río Páez, Cartago.

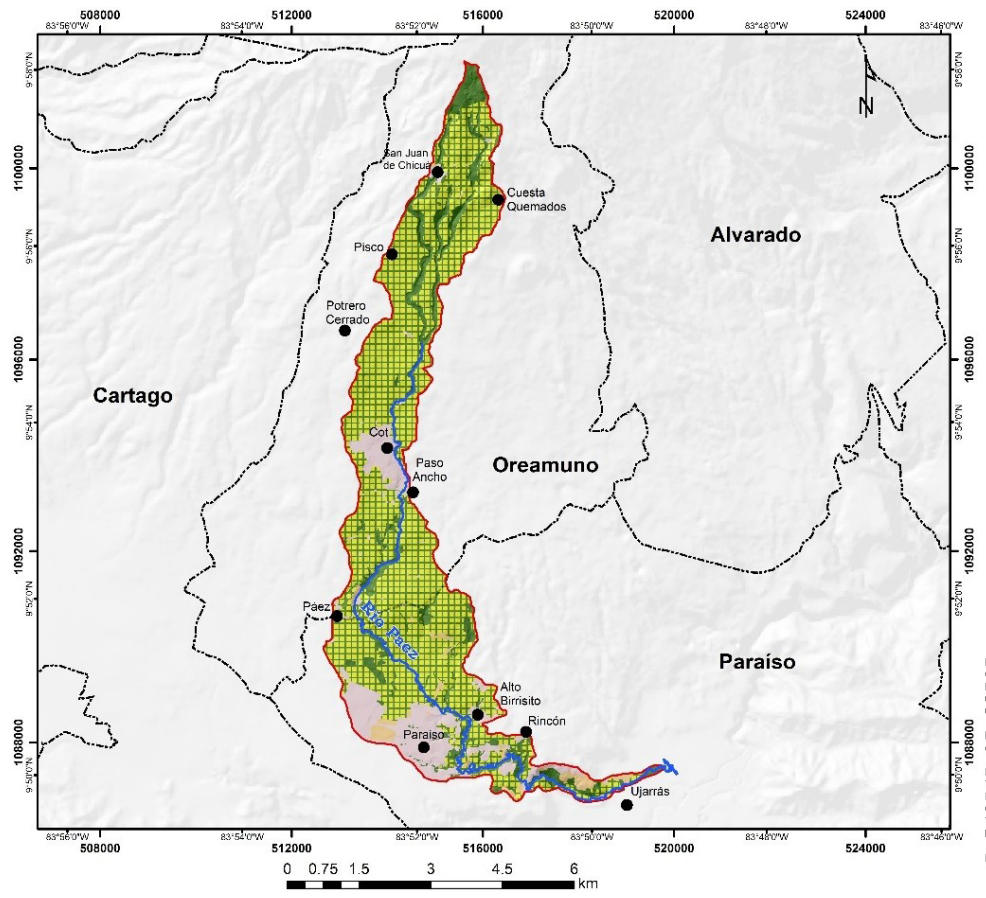

$$
\begin{aligned}
& \text { Simbología } \\
& \text { Poblados } \\
& \text { Us Limite cantonal politico } \\
& \text { UsoActual del Suelo } \\
& \text { Bosque denso } \\
& \text { Zona urbana continua } \\
& \text { Mosaico de pastos y } \\
& \text { cultivos } \\
& \text { Otros cultivos } \\
& \text { permanentes }
\end{aligned}
$$

Diagrama de Ubicación

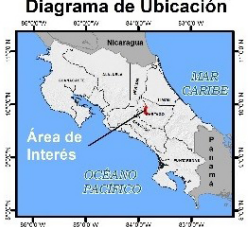

Fuente: Castro, 2020. 
No obstante, en zonas boscosas, se realizó aprovechamiento forestal en el período 2017-2018 en los distritos de Paraíso, Potrero Cerrado y Cot; de al menos 438 árboles (ciprés, eucalipto, pino y casuarina) correspondiente a un volumen de $864,355 \mathrm{~m}^{3}$. Además, se presentan denuncias interpuestas al MINAE por infracción ambiental; la mayoría relacionada con la deforestación (tala ilegal) infringiendo la Ley No. 7575 para el período 2017 y 2018 y más recurrente en la parte media y baja de la subcuenca en el sector de Paraíso de Cartago (B. Pavlotzky, comunicación personal, 15 julio del 2019) (cuadro 3).

\section{Principales amenazas}

El área de estudio forma parte de la red fluvial compuesta por ríos y quebradas considerados como puntos focales para el desarrollo de amenazas hidrometereológicas, en las cuales el río Páez forma parte (CNE, 2008). Así mismo por encontrarse cerca del volcán existe amenaza de caída de cenizas en menor grado y exposición a piroclastos y se podrían afectar pueblos cercanos, los cultivos, la ganadería, y en el cauce del río Páez las corrientes de barro podrían provocar problemas. El 18\% de las personas consultadas indica que las inundaciones son una amenaza natural importante y se ve afectado en sitios impermeabilizados en la parte media y baja de la cuenca; por ejemplo, el poblado de Paso Ancho perteneciente al distrito de Cot ha sido una de las zonas más afectadas por las inundaciones.

Según CNE (2009), las inundaciones son más periódicas en el cantón de Oreamuno debido a la inadecuada planificación del territorio y mal manejo de desechos sólidos depositados en el cauce de los ríos. Otra amenaza que presenta este sitio han sido los deslizamientos o inestabilidad del terreno; los poblados más vulnerables son Cot, Paso Ancho y San Juan de Chicúa, y esto se debe a las características de fuertes pendientes en la parte alta y media de la subcuenca del río Páez (CNE, 2009).

\section{Biodiversidad}

La subcuenca del río Páez se encuentra ubicada dentro del Área de Conservación Cordillera Volcánica Central; en ella está el Parque Nacional Volcán Irazú, que abarca parte del cantón de Oreamuno. 
La biodiversidad que existe en las subcuencas Birris y Páez pertenece a la vegetación tipo páramo y se encuentra compuesta por hierbas y arbustos de hojas pequeñas de baja estatura.

Según un censo realizado por Alfaro (2017) existen aproximadamente 252 especies de árboles en el distrito de Cot y Paraíso evidenciando una diversidad importante de especies.

En la parte alta de la subcuenca del río Páez perteneciente a la zona de vida Bosque muy húmedo Montano (BmhM), se ubica el sector de Prusia donde nacen los ríos Reventado y Páez, presenta un bioclima limitante para muchas actividades de uso del suelo debido a su baja temperatura parte media está compuesta por el Bosque Húmedo Montano Bajo (Bh-MB) y la parte baja de la subcuenca presente el Bosque Húmedo Premontano (SINAC, 2008).

Las especies forestales de cedro amargo, aguacatillo, sauce, cristóbal, níspero, zapote, robles, cedrillo, caobilla, gavilán y jaúl se encuentran en la parte baja de la subcuenca (INDER, 2016).

Respecto a la fauna hay águilas, halcones, búhos, gallina de monte, conejos, paloma de monte, cuyeo, taltuza, quetzal, ardillas, zorro pelón, ratón de monte, perezosos de dos y tres dedos, coyote, mapaches, pizotes, serpientes de altura y salamandras (Artavia, 2017).

El territorio Paraíso-Alvarado es un sector considerado como una de las regiones de mayor diversidad biológica y se han descubierto gran cantidad de especies endémicas por su ubicación geográfica porque protege gran variedad de ecosistemas como: páramos, turberas, ciénegas, sabanas no arboladas, bosques de jaúl y bosques nubosos constituidos estos últimos por robledales de altura (INDER, 2016).

Para el periodo 2019 el área de estudio cuenta con 501 hectáreas dedicadas a Pago por Servicios Ambientales (PSA) en la modalidad de Protección del recurso hídrico y Sistemas Agroforestales (SAF). Estas áreas brindan servicios hídricos y contribuyen a la cuenca para asegurar disponibilidad de agua, proteger la cobertura boscosa, y tener sistemas productivos con diferentes propósitos de producción (J. Hernández, comunicación personal, 24 junio del 2019). 


\section{Aspectos socioeconómicos}

La demografía de la subcuenca del río Páez está constituida por 35126 habitantes asentados en los pueblos de Oreamuno y Paraíso (INEC, 2011) distribuidos en 11 poblados. Se cuenta con 12 centros educativos escuelas, colegios diurnos y nocturno, e institutos. El cantón de Paraíso tiene una población alfabeta de 98\% y Oreamuno con 97.9\% (Atlas del Desarrollo Humano Cantonal de Costa Rica, 2016). Los habitantes de la cuenca poseen un nivel de escolaridad adecuado; el $60 \%$ de la población poseen primaria completa, $22 \%$ corresponde a educación secundaria completa y educación superior. Los pueblos de Paraíso, Paso Ancho y Ujarrás reportan mayores niveles de escolaridad y los centros educativos son apropiados.

Paralelamente tienen programas de educación ambiental, por ejemplo, en el periodo 2017-2019 se realizaron 68 actividades en el Cantón de Oreamuno y Paraíso a cargo del SINAC. El 49\% de las personas consultadas indican que se realizan campañas de reciclaje y un $51 \%$ menciona que no hay y que lo desconoce, aun así, se deben fortalecer dichos programas en el ámbito formal y no formal, ya que la subcuenca presenta problemáticas asociadas a aspectos socioeconómicos y que pueden ser promovidas a través de la educación ambiental.

Respecto al servicio de salud, las comunidades cuentan con adecuados centros de salud; el $89 \%$ de la población tiene cobertura de seguro social, y tan solo el 11\% no lo están; estas personas pertenecen a las comunidades de Cot y Paso Ancho. En la parte alta de la cuenca hay menos incidencia a enfermedades frecuentes y en la parte media y baja presentan enfermedades más frecuentes son las respiratorias, que abarcan un $54 \%$ de la población y un $23 \%$ corresponde a problemas de diarreas, específicamente en Paraíso, Paso Ancho y Ujarrás. Por tanto, estas poblaciones tienen más incidencia a problemas de contaminación del rio y afectación a la salud de personas.

En relación con la infraestructura física o de viviendas, en la parte alta y media, del $100 \%$ de los habitantes, el $94.5 \%$ poseen casa y $4.5 \%$ choza o rancho, de los cuales el $77 \%$ tiene vivienda propia y un $23 \%$ son alquiladas o prestadas. En términos de las características de las viviendas en su mayoría son de concreto equivalente al $84 \%$, y el resto son construidas a base de madera, zinc u otro material; y en la parte baja el sector de Paraíso es el que tiene la mayor cantidad de viviendas 5870 a nivel rural y 
nivel urbano. Por lo planteado se evidencia apropiadas condiciones de las viviendas en la zona de estudio y esto representa que no hay condiciones precarias de las viviendas que puedan incidir en la calidad de vida de las personas por ende para la cuenca también.

Los servicios públicos se catalogan como adecuados por parte de las personas, el alcantarillado sanitario (58\%), recolección de basura $(85 \%)$, electricidad (83\%). Sin embargo, es necesario fortalecer el servicio de tratamiento de aguas negras y limpieza de calles por parte de los municipios y así degradar menos la calidad del agua del rio.

El $82 \%$ población tiene acceso al servicio de agua para consumo humano, la subcuenca tiene ríos y quebradas importantes para su abastecimiento, en ella se cuenta con 8 asadas y 2 acueductos municipales distribuidos en la subcuenca, lo cual se considera que suficientes los operadores de servicios de agua.

Se presenta una contaminación evidente en la subcuenca generada por el crecimiento de la población, uso de insumos en prácticas agrícolas, industriales-comerciales y las aguas residuales que se vierten de viviendas cercanas al cauce. Según Chin et al. (2016) el río Páez presenta contaminación de desechos sólidos en las orillas y en el cauce, como envases vacíos de agroquímico, plásticos, telas y prendas de ropa, presencia y generación de grandes cantidades espuma en el agua, lo que hace suponer la presencia de agentes surfactantes, como detergentes en el agua.

Dicha contaminación está afectando los ecosistemas hídricos, la vida acuática y la calidad del agua específicamente en la parte media y baja de la subcuenca (Figura 7), y se requiere estrategias de saneamiento ambiental para el tratar aguas negras provenientes de viviendas, industria y actividades agropecuarias y disminuir el deterioro de la calidad del agua del rio Páez. 
Figura 7. Problemas de contaminación Cot, parte media de la subcuenca del rio Páez, Cartago.

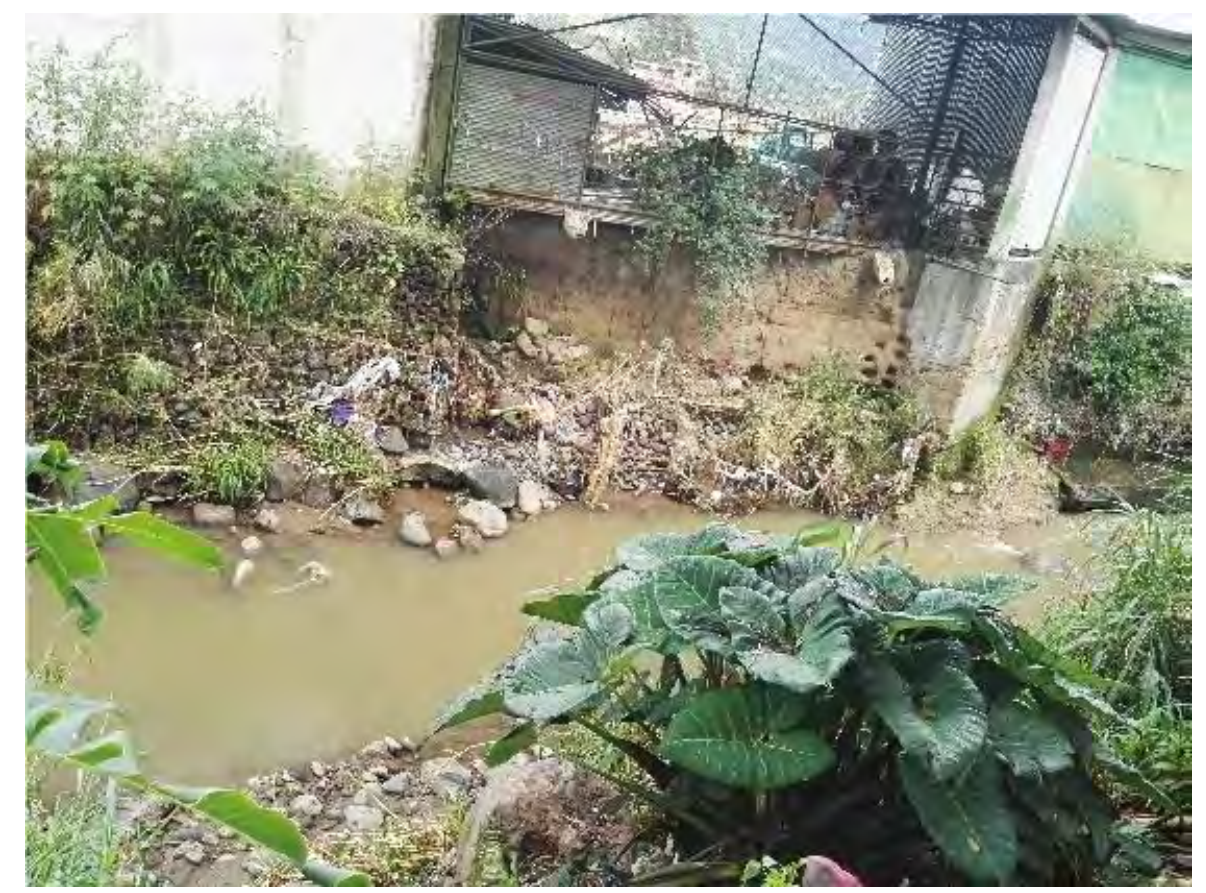

Fuente: M. Álvarez, 2018

En relación con la infraestructura vial y de transporte, INDER (2016) menciona que la red vial del sector de Oreamuno (parte alta y media de la cuenca) es fluida, el transporte público comunica a todos los cantones para la población se movilice a Cartago a recibir distintos servicios, así mismo el $73 \%$ de la población considera apropiado el servicio de transporte público. En la parte media y baja (cantón de Paraíso) la infraestructura vial y la conectividad entre los lugares es aceptable, pero requiere mejorar los caminos y carreteras, tanto, así como la señalización y alumbrado público.

El tema del empleo en la subcuenca plantea que el $40 \%$ población se dedica a labores en el sector educación, instituciones del estado, servicios profesionales, industria, administración y un gran sector que se encuentra pensionados. Así mismo un 25\% labora en la agricultura, $24.72 \%$ al sector comercio (Paraíso) y el resto a actividades ganaderas, construcción y oficios domésticos. 
De acuerdo con MIDEPLAN (2018) el cantón de Oreamuno ocupo en el periodo 2017 un índice de desarrollo social de 54.71 representando un área de menor desarrollo, y Paraíso obtuvo 56.82, correspondiente un desarrollo medio. Adicionalmente los distritos ubicados en la parte alta y media se clasifican en el nivel bajo como Potrero Cerrado (56.25) y Cot (60.2), principalmente por ser comunidades en las cuales sus habitantes cuentan con poca o nula fuente de empleo, luego Paraíso se ubica posee un nivel medio (73.9).

Paralelamente la gobernabilidad local del área de la cuenca está representada por los gobiernos locales, y dentro de ellas han definido áreas estratégicas de desarrollo, una de ellas es el área ambiental. Sin embargo en el sector de Oreamuno el índice de Gestión Municipal Oreamuno (2017-2018), presenta una calificación de 68.15 y 66.30 , que evidencia que el eje de gestión de desarrollo ambiental debe fortalecerse, específicamente al adecuado depósito y tratamiento de los residuos sólidos y aseo de vías en sitios públicos, a su vez Paraíso obtuvo la calificación respectivamente 65.68 y 69.78, destacando también la necesidad de fortalecer el tema de aseo de vías y sitios públicos y parques, obras ornato, recolección residuos sólidos.

\section{Conclusiones}

La determinación de aspectos biofísicos y socioeconómicos muestra las condiciones actuales de la subcuenca del rio Páez, de las cuales se debe prestar atención, una de ellas es la actividad agropecuaria y ganadera que ocupa más del $50 \%$ del territorio de la cuenca y es una de las más desarrolladas, ubicada en sitios de pendientes pronunciadas utilizando prácticas no adecuadas provocando deterioro al suelo como lo es la erosión y contaminación en las fuentes de agua, por tanto se requiere implementar practicas sostenibles y apostar a una agricultura sostenible para disminuir el uso de agroquímicos y no afectar la salud humana y la calidad del agua del rio.

En los poblados de la cuenca (Cuesta los Quemados, Pisco, Cot, Alto Birrisito, Paso Ancho y San Juan de Chicúa), poseen terrenos vulnerables a deslizamientos debido a las características de pendientes fuertes (más $30 \%$ ) propiciando procesos de erosión y degradación de la cuenca, por tanto, el mantenimiento de la cobertura forestal (como segundo uso $15 \%$ ) es fundamental para la captación hídrica, belleza escénica para el turismo y para ampliar más contratos Pago por Servicios Ambientales, acompañado 
por la implementación de Sistemas Agroforestales en las fincas para aumentar la cobertura forestal.

El rio Páez muestra riqueza hídrica y es una fuente importante de agua potable para abastecer sectores de Oreamuno y la ciudad de Cartago, también para generar energía eléctrica y favorecer el desarrollo de actividades agrícolas, pecuarias e industriales.

Así mismo en cuanto al saneamiento del agua se requiere una gestión permanente para el tratamiento de las aguas negras y disminuir el deterioro de la calidad del agua del rio, alineado la diversificación de programas ambientales (educación formal y no formal) en los poblados que contemplan temáticas más allá de programas de reciclaje, y que puedan incluir alfabetización hídrica, diseño de propuesta educación ambiental orientada a la gestión integrada del manejo de cuencas hidrográficas, saneamiento de las aguas en los ríos como una estrategia para educar a la población sobre el agua y el saneamiento en la subcuenca

Diversificar las actividades económicas para generación de empleo en la zona, como la agricultura más sostenible, crear emprendimientos exitosos (agricultura orgánica, turismo rural sostenible, etc.) y poder tener acceso a créditos para financiar otras actividades y contar con más apoyo por parte de entes estatales. Finalmente, a través del estudio biofísico y socioeconómico, vasto para conocer las condiciones y la realidad en la que se encuentra la subcuenca del rio Páez y que son necesarias para proponer alternativas de manejo.

\section{Referencias}

Alfaro, K. (2018). Zonificación de infraestructuras verdes para reducir el volumen de escorrentía y mitigar la vulnerabilidad a inundaciones, subcuenca río Páez, Cartago. (Tesis de licenciatura). Universidad Nacional, Costa Rica.

Alvarado, G. E., \& Vega, A. E. (2013). La geomorfología de la colada de Cervantes, Volcán Irazú (Costa Rica): descripción de uno de los campos de lava más grande de América Central. Revista Geológica de América Central, (48), pp. 99-118.

Arias, L. (1987) Diagnóstico y acciones propuestas para la protección de cuencas de acueductos municipales (Estudio piloto para trece municipalidades seleccionadas). CATIE. 
Osvaldo Navarro-Ceciliano

Estudio de capacidad de acogida del suelo en el Corredor Biológico Interurbano Río Torres, Costa Rica

Arias-Cascante, J. (2018). Mapeo de áreas prioritarias para la restauración ecológica en la zona de influencia del Centro Agrícola Cantonal de Oreamuno. Trabajo Final de Graduación (Licenciatura en Ingeniería Forestal) Instituto Tecnológico de Cartago Costa Rica, Escuela de Ingeniería Forestal. 106 p.

Artavia, I. (2017). Sistematización Cosechando agua pluvial, uniendo pueblos: La experiencia de la ASADA de Paso Ancho y Boquerón. ACCVC. Recuperado de http://www.sinac.go.cr/ES/partciudygober/Sistematizacion $\% 20$ Experiencias/INFORME $\% 208 \% 20-\% 20$ ASADA\%20PASO \%20ANCHO.pdf

Arellano, F., Vásquez, M., Suarez, J., y Espinoza, R. (2012). Informe Final: Identificación de zonas de protección y de captura de las $\mathrm{Na}$ cientes, subcuencas de los rios Reventado, Tiribi, Tatiscu, Páez, Birrís y Pacayas, provincia de Cartago, Costa Rica, p. 209.

Carrie, J. (2004). Manual de manejo de cuencas. El Salvador: Editorial World Vision.

Campos, C. (2010). Análisis de los cambios cobertura de la cuenca alta y media del Río Reventazón, Costa Rica. (Tesis de Licenciatura). Escuela de Ingeniería Forestal, Instituto Tecnológico de Costa Rica, Cartago, CR., p. 81.

Comisión Nacional de Emergencias [CNE] (2009). Descripción de amenazas del cantón de Oreamuno y Paraíso. Recuperado. https://www. cne.go.cr/reduccion_riesgo/mapas_amenzas/mapas_de_amaneza/ cartago/Oreamuno\%20-\%20descripcion\%20de\%20amenazas.pdf

Comisión Nacional de Emergencias. [CNE] (2009). Amenazas de origen natural cantón de Oreamuno. Recuperado https://www.cne.go.cr/reduccion_riesgo/mapas_amenzas/mapas_de_amaneza/cartago/Oreamuno\%20-\%20descripcion\%20de\%20amenazas.pdf

Comisión Nacional de Emergencias [CNE]. (2009). Amenazas de origen natural cantón de Paraíso. Recuperado https://www.cne.go.cr/reduccion_riesgo/mapas_amenzas/mapas_de_amaneza/cartago/Paraiso $\% 20-\overline{\%} 20$ descripcion $\% 20 \mathrm{de} \% 20$ amenazas.pdf

Coronel-Picón, Y. R., Obregón-Neira, N., \& Jiménez-Romero, G. L. (2012). Patrones de relación entre información biológica e información física y socioeconómica. Cuenca alta del río Otún, Risaralda (Colombia). Ingeniería y Universidad, 16(1), pp. 265-280. 
Chin, J; Aguilar, P, Vega, D. \& Espinoza, D. (2016). Descripción biofisica y resultados preliminares de la calidad del agua del área de la microcuenca del río Páez, asociada con el segmento del río comprendido entre su nacimiento y las zonas de protección de las nacientes Paso Ancho y Lankaster en la provincia de Cartago.CICA-UCR.57 p.

Elizondo, R. F. (2013). Los afluentes y los ríos: La construcción social del medio ambiente en la cuenca Lerma Chapala. ITESO. Retrieved from https://ebookcentral.proquest.com

INEC (Instituto Nacional de Estadística y Censos - CR). (2011). Censo 2011: Población total por zona y sexo, según provincia, cantón y distrito Recuperado https://www.inec.cr/documento/censo-2011-poblacion-total-por-zona-y-sexo-segun-provincia-canton-y-distrito

Instituto de Desarrollo Rural (INDER). (2016). Caracterización del Territorio: Cartago-Oreamuno-El Guarco- La Unión, pp. 1-38. Recuperado en https://www.inder.go.cr/correque/Caracterizacion-territorioCartago-Oreamuno-El-Guarco-La-Union.pdf

Instituto de Desarrollo Rural [INDER]. (2016). Caracterización del Territorio: Paraiso-Alvarado, p. 42. Recuperado en https://www.inder. go.cr/istaru/Caracterizacion-Territorio-Paraiso-Alvarado.pdf

Instituto Geográfico Nacional [IGN], Ministerio de Obras Públicas y Transportes [MOPT] (1981). Istarú. [Hoja topográfica]. Escala 1:50,000. San José, Edición 2.

Krushensky, R. (1972). Geology of the Istaru Quadrangle Costa Rica. Washington.: United States Department of the Interior. Geological Survey.Pirani, F. J., \& Mousavi, S. A. (2016). Integrating socio-economic and biophysical data to enhance watershed management and planning. Journal of hydrology, 540, pp. 727-735.

Ministerio de Planificación Nacional y Política Económica [MIDEPLAN]. (2018). Índice de desarrollo social 2017. San José, Costa Rica. Recuperado https://documentos.mideplan.go.cr/share/s/ L0GWBZnfRceDJjpxwm7zFQ

Programa de Investigación en Desarrollo Urbano Sostenible [PRODUS]. (2016). Plan del uso del suelo para las faldas del Volcán Irazú. San José: Universidad de Costa Rica, 177 pág.

Programa de las Naciones Unidas para el Desarrollo [PNUD]. (2016). Atlas del Desarrollo Humano Cantonal de Costa Rica Indicador de 
Osvaldo Navarro-Ceciliano

Estudio de capacidad de acogida del suelo en el Corredor Biológico Interurbano Río Torres, Costa Rica

Alfabetización de los cantones de Paraíso y Oreamuno. Recuperado en https://www.cr.undp.org/content/costarica/es/home/atlas-dedesarrollo-humano-cantonal.html

Programa Estado de la Nación. (2013). Indicadores Cantonales Censos Nacionales de Población y Vivienda 2000 y 2011. San José, Costa Rica: PEN. Recuperado en http://repositorio.conare.ac.cr/ handle/20.500.12337/882

Quesada, R. (2007). Los Bosques de Costa Rica. IX Congreso Nacional de Ciencias. Instituto Tecnológico de Costa Rica. Cartago, Costa Rica, 16. Ramírez, L., Alvarado, A., Pujol, R., \& Brenes, L. G. (2008). Caracterización Física de la Cuenca Media del Río Reventado, Cartago, Costa Rica. Agronomía Costarricense, 32(2), pp. 73-92.

Rojas, N. (2011). Cuenca del Río Reventazón. Instituto Meteorológico Nacional. Secretaría Ejecutiva de Planificación Sectorial Agropecuaria [SEPSA]. (2018). Estadísticas Agropecuarias. Recuperado en Inforagro: http:// www.infoagro.go.cr/EstadisticasAgropecuarias/Paginas/default.aspx Sistema Nacional de Áreas de Conservación [SINAC]. (2008). Plan General de Manejo Parque Nacional Volcán Irazú. Recuperado en http:// www.sinac.go.cr/ES/planmanejo/Plan\%20Manejo\%20ACC/Parque $\% 20$ Nacional $\% 20$ Volc $\% \mathrm{C} 3 \% \mathrm{~A} 1 \mathrm{n} \% 20 \mathrm{Iraz} \% \mathrm{C} 3 \% \mathrm{BA}$.pdf

Sistema Nacional de Áreas de Conservación [SINAC]. (2019). "Informe Anual Estadísticas SEMEC 2018: SINAC en Números”. Comp. B. Pavlotzky. San José, CR., p. 82.

Strahler, A. (1957). Quantitative analysis of watershed geomorphology. Transaction American Geophysical Union 38 (6), pp. 913-920.

Siles, J., \& Soares, D. (2003). La fuerza de la corriente: Gestión de cuencas hidrográficas con equidad de género. UICN. San José, CR, p. 266.

Villareal, N. S. (2007). Metodología, estrategia y aspectos técnicos en el manejo de conservación de cuencas. Biocenosis, 20(1-2), 1-7.

Watler, W., Faustino, J. y los miembros del Centro Agronómico Tropical de Investigación y Enseñanza. (2013, 1 de julio-30 de septiembre). Curso internacional en línea de especialización en "Gestión integral de cuencas hidrográficas": Construcción del Plan de Gestión de Cuencas Hidrográficas. (Unidad 2) [curso en línea]. CATIE. 
Osvaldo Navarro-Ceciliano Study of soil bearing capacity in the Rio Torres Interurban Biological Corridor, Costa Rica 